\title{
Influence of the Earth's ring current strength on Størmer's allowed and forbidden regions of charged particle motion
}

\author{
Alexander S. Lavrukhin, Igor I. Alexeev, and Ilya V. Tyutin \\ M.V. Lomonosov Moscow State University, Skobeltsyn Institute of Nuclear Physics (SINP MSU), 1(2), Leninskie gory, \\ GSP-1, Moscow 119991, Russian Federation
}

Correspondence: Alexander S. Lavrukhin (lavrukhin@physics.msu.ru)

Received: 9 September 2018 - Discussion started: 20 September 2018

Revised: 28 May 2019 - Accepted: 5 June 2019 - Published: 9 July 2019

\begin{abstract}
Størmer's particles' trapping regions for a planet with an intrinsic dipolar magnetic field are considered, taking into account the ring current which arises due to the trapped particles' drift for the case of the Earth. The influence of the ring current on the particle trapping regions' topology is investigated. It is shown that a critical strength of the ring current exists under which further expansion of the trapping region is no longer possible. Before reaching this limit, the dipole field, although deformed, retains two separated Størmer regions. After transition of critical magnitude, the trapping region opens up, and charged particles, which form the ring current, get the opportunity to leave it, thus decreasing the ring current strength. Numerical calculations have been performed for protons with typical energies of the Earth's radiation belt and ring current. For the Earth's case, the Dst index for the critical ring current strength is calculated.
\end{abstract}

\section{Introduction}

One of the first scientists who studied the problem of finding trajectories of charged particles moving from the Sun to the Earth was Norwegian physicist Carl Størmer. Since it is rather difficult to solve this problem directly without numerical methods, he used several simplifying assumptions: only a magnetic field of terrestrial origin (magnetic dipole) acts on a charged particle; magnetic and electric fields from other sources, including charged particle fluxes, are not taken into account (Størmer, 1955). In addition, the geometry of this problem was reduced to axial symmetry - the magnetic moment of the dipole is directed along the Earth's axis of rota- tion. As a result of the analysis, Størmer obtained the boundaries of the allowed regions of motion for particles coming to the Earth from infinity.

For a complete analytical solution of the problem, one has to find three integrals of motion. In the given problem the law of conservation of the generalized angular momentum of a particle follows from the isotropy of space (the Lagrangian does not change relative to the coordinate system rotations); the law of conservation of energy is the result of the fact that the Lagrangian does not change with respect to the time shift. The third integral of motion cannot be found analytically. Størmer solved this problem by obtaining an expression of a special parameter $\gamma$, which determines the geometry of allowed and forbidden regions of motion for a charged particle; particles with different energies can have the same $\gamma$ (see Sect. 3). Størmer's analysis has its main application in the prediction of the geomagnetic cutoff of cosmic rays. At the same time, the study of the topology of the charged particles motion regions is an interesting problem which allows us to understand the processes occurring in the Earth's magnetosphere at the global level.

The effect of the equatorial ring current on the intensity of cosmic rays was considered by Treiman (1953), where an attempt to determine the changes in the intensity of cosmic rays as a function of the ring current strength has been made. But the question of how the topology of particles' motion in the magnetosphere changes with growth of the current's strength has not been considered. This work is completely analytical, without quantitative estimates. Ray (1956) also carried out an analysis of the equatorial ring current influence on latitudinal changes in the intensity of cosmic rays. As a result, for the initial parameters of the cosmic ray spectrum, the ring 
current strength magnitude was obtained, which gave the observed latitudinal dependence of the vertical intensity of cosmic rays. The radius of the current ring was about 7.5 Earth radii, and the current strength was sufficient to create a magnetic field depression of $100 \mathrm{nT}$ at the geomagnetic equator. Regions of allowed particles motion have been studied in this paper only in connection with geomagnetic cutoff rigidity calculations. The expression for the current's vector potential was written for a filamentary ring current, because if the current ring has a finite cross section in this problem, this will greatly complicate it without noticeable change in the obtained results.

Also, Størmer's analyses have been expanded for the external homogeneous field case. Lemaire (2003) introduced a homogeneous and stationary field into the Størmer problem; the orientation of this field was parallel and antiparallel to the dipole magnetic moment. As a result, a new expression for Størmer's potential that takes into account the additional component of the external field was obtained. Lemaire (2003) showed how the inclusion of the northern or southern homogeneous fields affects the allowed and forbidden zones, and also that the southward direction enables easier access for solar energetic particles and galactic cosmic rays to the interiors of the geomagnetic field along the magnetic field lines, which connect the dipole with the interplanetary space.

The main sources which form the magnetospheric magnetic field are the internal field of the planet, interplanetary magnetic field (IMF) and the field of the magnetospheric current systems (Chapman-Ferraro currents at the magnetopause, magnetospheric tail currents and ring current). In the Størmer problem in connection with its specificity (axial symmetry), we will consider only the contributions of the dipole field (the contributions of the quadrupole and other multipoles have been considered in Shebalin, 2004, but only axially symmetric components, which are small for the Earth's case), the $b_{z}$ component of the external origin field (IMF or tail current sheet field) and field of an axially symmetric ring current. This approximation will allow us to estimate the influence of the ring current on the allowed regions of particle motion for the case of the Earth's magnetosphere. Also, we do not take into account the presence of a magnetopause located at a distance of several Earth radii on the dayside magnetosphere.

So far, Størmer's trapping regions have been considered separately from real trapping regions in the Earth's magnetosphere. We consider allowed regions of charged particles' motion in a wide energy range for different ring current strength magnitudes. We show that, using the Størmer analysis, one can find trapping regions for particles of the ring current and radiation belts energies, and also the fact that the forbidden region of particles' motion can break as the current increases, and then particles get an opportunity to leave the trapping region.

The present paper consists of the following sections: Sect. 2 describes the Earth's ring current and its general fea- tures; in Sect. 3 we consider the mathematical formulation of the Størmer problem; in Sect. 4 we consider the effect of Størmer's parameter $\gamma$ change on the allowed-forbidden regions' configuration; in Sect. 5 the trapping regions are modeled with different magnitudes of the ring current strength.

\section{The Earth's ring current}

The dipole configuration of the Earth's magnetic field creates a region that is a kind of a "magnetic vessel" inside which charged particles are trapped. For the first time the possibility of the existence of such a region was shown by Carl Størmer (Størmer, 1912). In accordance with the energy, the trapped particles can be divided into three groups: the radiation belt, the ring current and the plasmasphere. The trapping regions in which the particles of each group are contained partially overlap. The energy of the radiation belt ions is between 1 and $100 \mathrm{MeV}$; they occupy the shells with $L \approx 1.2-$ 2.5 (Prölss, 2004). The energy of the ring current ions is between 1 and $200 \mathrm{keV}$ (Prölss, 2004); it occupies an area with $L \approx 2.3-7.8$ (Kovtyukh and Panasyuk, 2008).

For the first time the hypothesis of a ring current was made by Carl Størmer to explain the location of the aurora and its shift to the equatorial latitudes under magnetic storm conditions (Størmer, 1912). He considered the influence of the ring current located in the Earth's equatorial plane on the structure of the magnetic field and the trajectories of electrons. Størmer showed that if the ring current could create a disturbance of $300 \mathrm{nT}$ on the equatorial surface of the Earth, then the aurora would move to the magnetic latitude of Christiania (district in Copenhagen) (Størmer, 1912).

Magnetic storms on the Earth are directly related to the ring current. The increase in its strength leads to compression of the inner magnetic field of the planet. During quiet times, the ring current is localized near the geomagnetic equator, directed to the west, and depends little on local time. A geomagnetic storm usually begins with the impact of a strong and permanent southward-oriented IMF along with the increased dynamic pressure of the solar wind on the dayside magnetosphere. The increased pressure moves the magnetopause inward by several Earth radii, increasing currents on the magnetopause and temporarily causing an increase in the surface magnetic field. This phenomenon is known as the sudden commencement of a storm. During the main phase of the magnetic storm, which is usually associated with the southward-oriented IMF magnitude growth, the electric field applied to the magnetosphere increases, which leads to an increase in the number of particles injected into the inner magnetosphere due to the $\boldsymbol{E} \times \boldsymbol{B}$ drift from the magnetotail. These particles significantly increase the quiet ring current, eventually creating a so-called storm-time ring current. This leads to a strong Dst index (axisymmetric component of the disturbed magnetic field relative to the geomagnetic dipole) increase and marks the main phase of the storm. The main 
phase generally lasts for several hours, but for specific storms its duration can vary greatly. After the IMF changes its direction from southward to northward, the recovery phase begins, during which the injection of the plasma layer material slows down or almost stops and the various loss processes return the ring current to its quiet state. The dominant source of the ring current is a plasma sheet, so the two most important driving parameters for the storm-time ring current are the near-Earth plasma sheet ion characteristics and the convection electric field intensity (Kozyra and Liemohn, 2003).

The strength of the storm-time current is estimated by the Dst index and can reach 10 MA (Baumjohann et al., 2010). For comparison, the magnitude of the region 1 longitudinal currents, which transfer the energy of the solar wind plasma to the magnetosphere under quiet conditions, is $1-3 \mathrm{MA}$, and the currents at the magnetopause are approximately 2 times larger: 2-6 MA (Alexeev, 2003). A distinctive feature of the storm-time ring current in comparison with the quiet-time ring current is its pronounced asymmetry. On the nightside, the strength of the storm-time current is much larger than on the dayside. Radial flow and pressure profiles of ring current particles during quiet periods have a maximum at $L_{\max }=5.5-6.0$, and during storms at $L_{\max }=2-5$ (Kovtyukh and Panasyuk, 2008). In quiet times, the current mainly consists of protons (average partial concentration $\sim 90 \%$, Daglis et al., 1999). During the magnetic storms there is a sharp increase in the density of atomic oxygen ions $\mathrm{O}^{+}$originating from the ionosphere. However, protons continue to make the main contribution to the ring current during typical storms, with an average partial energy density of $\sim 70 \%$ (Daglis et al., 1999). During very large storms, the $\mathrm{O}^{+}$ion component becomes predominant (Daglis et al., 1999).

During the main phase of the storm, the hot plasma is injected into the inner magnetosphere. The stronger the storm, the closer the ring current comes to the Earth and the slower it relaxes. Storm-time ring current essentially differs from radiation belts, consisting of more energetic particles, in composition, dynamics and generation mechanisms. Unlike radiation belt ions, whose fluxes are weakened during storms (Kovtyukh and Panasyuk, 2008), the fluxes of particles of the ring current increase by 1 to 3 orders of magnitude during the main phase of the storms, and after the recovery phase they decrease to some quasistationary levels.

The ring current is not the only source which contributes to the Dst index during the storm. Alexeev et al. (1996) and Turner et al. (2000) showed that the fields created by the magnetotail currents during the main phase of the storm contribute $25 \%$ of Dst, the amount of which, however, significantly decreases during the recovery phase. The magnetic effects of the magnetotail together with the asymmetric ring current give the largest contribution during the main phase (Alexeev et al., 1996; Turner et al., 2000).

There are several loss processes which act on decay of the ring current ions: the charge exchange of ions with the exospheric atoms, the Coulomb drag, the Coulomb scattering, pitch angle scattering due to interaction with electromagnetic waves, the violation of the first adiabatic invariant and the convection outflow. All these sources are considered in detail in Ebihara and Ejiri (2003). The outflow of ions through the dayside magnetopause can be a significant term in the energy balance of the storm-time ring current during the main phase of the storm (Kozyra and Liemohn, 2003). Also, the initial fast stage of the recovery phase of very strong storms can be associated with the decay of the oxygen component, and the subsequent slow stage with the decay of the proton component (Daglis et al., 1999). Rapid decay of the inner part of the magnetospheric tail current (Alexeev et al., 1996) and the sudden change in the IMF direction to northward at the end of the main phase of the storm were also considered mechanisms which act during the fast phase of the recovery of powerful storms, which can lead to an inversion of the convection electric field and ejection of the ring current from the outer regions of the trapping region (Ebihara and Ejiri, 1998).

We propose a new loss mechanism associated with the disappearance of Størmer's forbidden region of particle motion between two allowed regions (outer, which is connected with the outer space, and inner, which is not) due to an increase in the ring current strength. This consideration allows us to find the upper limit on the maximum current, which can be created by trapped particles. In this threshold case, the ring current particles are allowed to leave the trapping region, which leads to a weakening of the ring current and Dst index decrease. The existing mechanisms of scattering and precipitation of particles can lead to the fact that the equilibrium value of the ring current will be smaller (in the case of the Earth by more than an order of magnitude) than this threshold value. But can this mechanism limit the maximum field depression during large magnetic storms?

In the zeroth approximation, the ring current can be represented as a ring with current in the equatorial plane at a certain distance from the Earth. The magnetic field in the center of the ring with a current of radius $R$ is given by Landau et al. (1982):

$B=\frac{2 \pi I}{c R}$.

From this expression, we can obtain an approximation of the magnetic field perturbation in the Earth's center:

$\Delta B_{\mathrm{rc}}=-\frac{2 \pi \Delta I_{\mathrm{rc}}}{c L R_{E}}$,

where $R=L R_{E}$ is the geocentric distance and $\Delta I_{\mathrm{rc}}$ is the increase in the ring current strength. This description is only approximate: in reality the ring current has a much more complex structure. However, this approach can be used for understanding the processes which occur at changes in the ring current strength and for estimation of the maximum number of particles which may be trapped by the dipole field. 
Based on the data given in this chapter, we will consider the distance to the ring current from the Earth's center to be equal to $a=4 R_{\mathrm{E}}$. In connection with the axial symmetry of the Størmer problem, only the symmetric ring current in the form of an infinitely thin ring will be used.

\section{The mathematical formulation of the Størmer problem}

Equations of the charged particle motion in an electromagnetic field in the case of axial symmetry are more convenient to consider in cylindrical coordinates. We consider a cylindrical coordinate system in which the positive $z$ axis is directed to the north of the planet. The dipole moment $M$ is located at the origin of coordinates and is directed along the negative direction of the $z$ axis; the external magnetic field has only the $z$ component. The radius of the planet is denoted as $R_{\mathrm{E}}$. The radius of the ring with current is denoted as $a$, the ring is located in the magnetic equatorial plane perpendicular to the $z$ axis, and the origin of coordinates lies in the plane of the ring in its center. In this case, we can speak about the axial symmetry of the magnetic field configuration, since the fields of the dipole and ring current and external field have axial symmetry in this system. We also use the following assumptions used by Carl Størmer: the magnetic field is stationary; we neglect the particle radiation as it moves in a magnetic field.

We will use the Lagrangian formulation; the equation of motion can then be written as (Landau and Lifshitz, 1976)

$\frac{\mathrm{d}}{\mathrm{d} t} \frac{\partial L}{\partial \dot{q}_{i}}-\frac{\partial L}{\partial q_{i}}=0$

The Lagrangian function $L$ of a charged particle in the relativistic case is (Morozov and Soloviyov, 1963)

$L=-m c^{2} \sqrt{1-\dot{r}^{2} / c^{2}}+\frac{Z e}{c}(\dot{\boldsymbol{r}} \cdot \boldsymbol{A}(\boldsymbol{r}, t))-e \Phi(\boldsymbol{r}, t)$,

where $\dot{\boldsymbol{r}}=(\dot{\rho}, \rho \dot{\boldsymbol{\phi}}, \dot{z})$ is the particle's velocity, $\boldsymbol{A}(\boldsymbol{r}, t)$ and $\Phi(\boldsymbol{r}, t)$ are vector and scalar potentials of an electromagnetic field, $Z$ is the charge of a particle in units of an electron charge, $m$ is the mass of a particle, and $c$ is the speed of light.

The scalar potential of an electromagnetic field will not be taken into account due to the absence of an electric field in Størmer's problem. Since the field we are considering has the axial symmetry, in a cylindrical coordinate system the Lagrangian does not depend on the azimuth angle $\phi$, and hence, by Noether's theorem, the generalized angular momentum (the first integral of motion) is conserved along the trajectory:

$P_{\phi}=\frac{\partial L}{\partial \dot{\phi}}=\frac{m \rho^{2} \dot{\phi}}{\sqrt{1-\dot{r}^{2} / c^{2}}}+\frac{Z e}{c} \rho A_{\phi}=$ const.
The total energy of a particle in the Lagrangian formulation is

$H=\dot{r} \frac{\partial L}{\partial \dot{r}}-L=\frac{m c^{2}}{\sqrt{1-\dot{r}^{2} / c^{2}}}$.

Since we neglect the particle radiation, the total energy $H$ in the constant magnetic field is conserved, and therefore, from Eq. (4) we get

$\dot{r}^{2}=v^{2}=$ const.

In other words, the square of the velocity of the particle $v^{2}$ is also a constant in the absence of the electric field (the second integral of motion). We rewrite Eq. (5) using velocity components in cylindrical coordinates:

$\left(\frac{\mathrm{d} \rho}{\mathrm{d} t}\right)^{2}+\left(\rho \frac{\mathrm{d} \phi}{\mathrm{d} t}\right)^{2}+\left(\frac{\mathrm{d} z}{\mathrm{~d} t}\right)^{2}=v^{2}$.

It is convenient to integrate over the arc of the trajectory $s(\mathrm{~d} s=v \mathrm{~d} t)$ instead of integrating over time $t$ and then to divide Eq. (6) by $v^{2}$ :

$\left(\frac{\mathrm{d} \rho}{\mathrm{d} s}\right)^{2}+\left(\rho \frac{\mathrm{d} \phi}{\mathrm{d} s}\right)^{2}+\left(\frac{\mathrm{d} z}{\mathrm{~d} s}\right)^{2}=1$.

We express $\dot{\phi}$ from Eq. (3) and substitute the square root from Eq. (4):

$\rho \dot{\phi}=\frac{c^{2}}{H}\left(\frac{P_{\phi}}{\rho}-\frac{Z e A_{\phi}}{c}\right)$.

From the relativistic relations, we can obtain the relation $p c^{2}=v H$, where $p$ is the particle's momentum; then we express $c^{2} / H=v / p$ and proceed from integration over $\mathrm{d} t$ to integration over $\mathrm{d} s$; finally we obtain

$\rho \frac{\mathrm{d} \phi}{\mathrm{d} s}=\frac{v_{\phi}}{v}=\frac{P_{\phi}}{p \rho}-\frac{Z e A_{\phi}}{p c}$.

After substituting this relation into Eq. (7),

$\left(\frac{\mathrm{d} \rho}{\mathrm{d} s}\right)^{2}+\left(\frac{\mathrm{d} z}{\mathrm{~d} s}\right)^{2}+\left(\frac{P_{\phi}}{p \rho}-\frac{Z e A_{\phi}}{p c}\right)^{2}=1$.

For clarity, we rewrite this equation using the expression from Eq. (8):

$$
\begin{aligned}
\left(\frac{\mathrm{d} \rho}{\mathrm{d} s}\right)^{2}+\left(\frac{\mathrm{d} z}{\mathrm{~d} s}\right)^{2} & =Q=1-\left(\frac{P_{\phi}}{p \rho}-\frac{Z e A_{\phi}}{p c}\right)^{2} \\
& =1-\left(\frac{v_{\phi}}{v}\right)^{2}
\end{aligned}
$$

where $Q$ is the kinetic energy of particle motion in the meridional plane (Størmer, 1955).

As can be seen from Eq. (10), during the motion of the particle, the condition $0 \leq\left(v_{\phi} / v\right)^{2} \leq 1$ must be satisfied, so 
that the motion of a charged particle in an axially symmetric magnetic field takes place in regions of the meridian plane for which condition $-1 \leq v_{\phi} / v \leq 1$ is met - these are the allowed regions of the particle's motion. The regions for which $\left(v_{\phi} / v\right)^{2}>1$ are forbidden. The boundary between these regions is determined by the equality of the velocity component $v_{\phi}$ and the velocity of the particle $v$; at this moment the particle turns around on the boundary and starts to move in the opposite direction. The existence of the boundaries of charged particle motion regions allows one to make a qualitative analysis of their motion for the chosen $A_{\phi}(\rho, z)$. In terms of energy, the minimum value of the fraction of kinetic energy $Q$, associated with motion in the meridional plane $(\rho-z)$, is equal to 0 ; at this moment the velocity of the particle is perpendicular to the meridian plane and the projection of the velocity on the $(\rho-z)$ plane changes sign; i.e., the particle, after reaching the point where $Q=0$, turns back. Therefore, the line $Q=0$ in the $(\rho-z)$ plane limits the region, inaccessible to particles.

Under these conditions, trapping regions, separated from infinity by forbidden regions (where particles cannot get due to the preservation of exact integrals of motion for any initial conditions), are formed in the phase space. Depending on the initial conditions, the trajectories of particles of a given energy may have a scattering pattern - particles come from infinity, change their direction of motion near the dipole, and again go to infinity, deviating by the scattering angle, or if their position and the generalized angular momentum satisfy Størmer's conditions, the particles make a finite motion, filling the allowed Størmer regions and forming the trapped particles' fluxes. This moment is given little attention in Størmer's original work (Størmer, 1955), who was mainly interested in the calculation of the penetration regions of cosmic ray particles, i.e., only those particles that come from infinity.

The surface $Q=0$, which separates the allowed and forbidden regions of motion, is determined from Eq. (10):

$Q=1-\left(\frac{P_{\phi}}{p \rho}-\frac{Z e A_{\phi}}{p c}\right)^{2}$.

The vector potential of the magnetic field produced by a magnetic dipole with moment $M$ is given by

$A_{\phi d}=\frac{M \rho}{r^{3}}$.

The vector potential of a homogeneous magnetic field $b_{z}$, directed along the $z$ axis (in our case this field characterizes the $z$ component of the external field, which may be either IMF or the magnetotail currents' field) is given by (Lemaire, 2003)

$A_{\phi e}=\frac{b_{z} \rho}{2}$.

The vector potential of the ring current has a complex form and depends on the assumed radius of its cross section. In our problem we assume that the ring current has the form of a ring with radius $a$ lying in the equatorial plane with the center at the origin, the cross section of which is much smaller than a. In this case,

$A_{\phi r}=-\frac{\pi I a}{c} \sum_{n=0}^{\infty} \frac{(-1)^{n}(2 n-1) ! !}{2^{n}(n+1) !} \frac{r_{<}^{2 n+1}}{r_{>}^{2 n+2}} P_{2 n+1}^{1}(\rho / r)$,

where $(2 n-1) ! !=(2 n-1)(2 n-3) \ldots 5 \cdot 3 \cdot 1$ and the coefficient for the term with $\mathrm{n}=0$ by definition equals $1 ; r_{<}$and $r_{>}$are, respectively, the smaller and larger of $a$ and $r ; P_{2 n+1}^{1}$ are the associated Legendre polynomials (Jackson, 1963).

One can separate the considered region into two parts: when $\rho<a$ and when $\rho>a$. Then the vector potential (Eq. 14) will be

$$
\begin{aligned}
A_{\phi r} & =-\frac{\pi I}{c} \sum_{n=0}^{\infty} \frac{(-1)^{n}(2 n-1) ! !}{2^{n}(n+1) !} \\
& \cdot P_{2 n+1}^{1}(\rho / r) \cdot \begin{cases}(\rho / a)^{2 n+1}, & \rho<a, \\
(a / \rho)^{2 n+2}, & \rho>a .\end{cases}
\end{aligned}
$$

Substituting all expressions for $A_{\phi}$, Eqs. (12), (13), and (14) of the vector potential, in Eq. (11), we obtain

$$
\begin{gathered}
1-\left(\frac{P_{\phi}}{p \rho}-\frac{Z e}{p c}\left(\frac{M \rho}{r^{3}}+\frac{b_{z} \rho}{2}-\frac{\pi I}{c} \sum_{n=0}^{\infty} \frac{(-1)^{n}(2 n-1) ! !}{2^{n}(n+1) !}\right.\right. \\
\left.\left.\cdot\left\{\begin{array}{ll}
(\rho / a)^{2 n+1}, & \rho<a \\
(a / \rho)^{2 n+2}, & \rho>a
\end{array}\right\} P_{2 n+1}^{1}(\rho / r)\right)\right)^{2}=0 .
\end{gathered}
$$

We define the Størmer radius $r_{\mathrm{S}}$ (Størmer, 1955), the Størmer parameter $\gamma$, and the dimensionless variable $R$ as

$r_{\mathrm{s}}=\sqrt{\frac{Z e M}{p c}} ; \gamma=\frac{P_{\phi}}{2 p r_{\mathrm{s}}} ; R=\frac{r}{r_{\mathrm{s}}}$.

In the case of the Earth, $r_{\mathrm{S}}$ dependence on the particle's energy is shown in Fig. 1. The higher the energy of the particle is, the smaller its Størmer radius is.

Størmer's parameter $\gamma$ is the ratio of the azimuthal components of two different momenta of the particle: $P_{\phi}$, taken at infinity, and the product of momentum $p$ and Størmer radius $r_{\mathrm{s}}$. This ratio is used with a multiplying factor of $1 / 2$ (taken for convenience of demonstration), $\gamma=0.5 P_{\phi} / p r_{\mathrm{s}}$; particles of any energy can have the same $\gamma$. In the case of the negatively charged particles $(Z e<0)$, the azimuthal component of the generalized momentum is directed to the west $\left(P_{\phi}<0\right)$, and, respectively, $\gamma<0$. In the case of the positively charged particles $(Z e>0)$, the azimuthal component of the generalized momentum is directed to the east $\left(P_{\phi}>0\right)$, and, respectively, $\gamma>0$. In our problem we will consider only protons and therefore gamma values greater than zero.

Having two integrals of motion and the Størmer parameter $\gamma$, one cannot completely describe the trajectory of any particle, but can find the regions of allowed motion and, thus, 


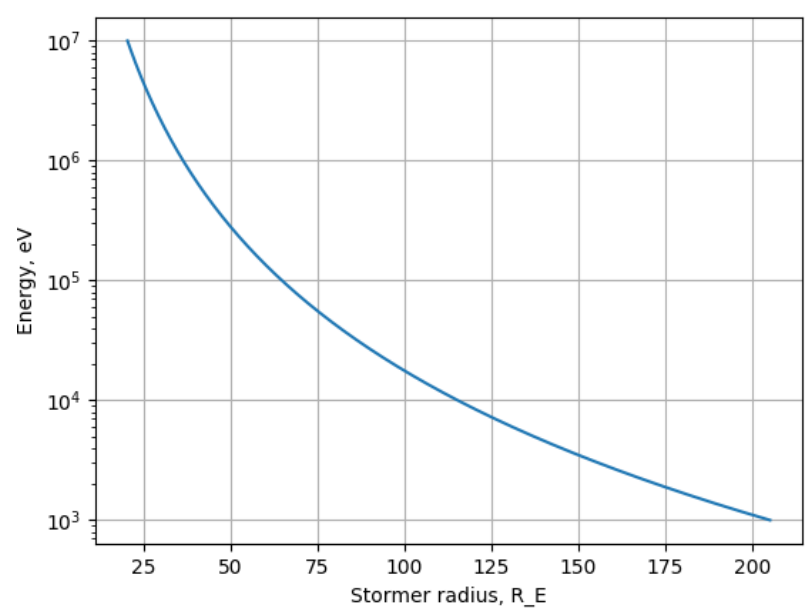

Figure 1. Dependence of the Størmer radius of the particle (in Earth radii) on its energy.

the trapping regions. We rewrite Eq. (11) taking into account the newly introduced quantities and expressing the distance in Størmer units:

$Q=1-\left(\frac{2 \gamma}{\rho}-\frac{Z e A_{\phi}}{p c}\right)^{2}$

The size of the allowed regions of motion, as can be seen from Eq. (18), will be different for particles with different energies and with different $\gamma$. The transition to the Størmer units of length allows one to analyze the motion of particles of different energies uniformly.

Using Eq. (17), we can rewrite Eq. (16) in dimensionless form, where the units of length are expressed in Størmer units of length:

$$
\begin{gathered}
1-\left(\frac{2 \gamma}{\rho}-\frac{\rho}{r^{3}}-\frac{b_{z} \rho}{2 M} r_{\mathrm{s}}^{3}+\frac{\pi I}{M c} r_{\mathrm{s}}^{2} \sum_{n=0}^{\infty} \frac{(-1)^{n}(2 n-1) ! !}{2^{n}(n+1) !}\right. \\
\left..\left\{\begin{array}{ll}
(\rho / a)^{2 n+1}, & \rho<a \\
(a / \rho)^{2 n+2}, & \rho>a
\end{array}\right\} P_{2 n+1}^{1}(\rho / r)\right)^{2}=0 .
\end{gathered}
$$

After solving Eq. (19), one can find the boundaries of Størmer's allowed and forbidden regions of the particles' motion for the general case of the sum of a dipole field, an external field and a ring current field.

\section{Størmer's parameter $\gamma$ properties}

As mentioned above, the allowed regions of a particle's motion are determined by the $-1 \leq v_{\phi} / v \leq 1$ condition. On the nearest and farthest boundaries to the Earth of the allowed region $\left|v_{\phi}\right|=v$, the particle changes its direction of motion to the opposite, and $\left|v_{\phi}\right|$ decreases and at some point goes to zero. Let us write Eq. (8) for this case:

$\frac{v_{\phi}}{v}=\frac{P_{\phi}}{p \rho}-\frac{Z e A_{\phi}}{p c}=0$.
Using Eq. (17), one can obtain

$2 \gamma=\frac{Z e}{p c} \rho A_{\phi}$.

In our problem $\gamma$ is constant, which is set as an initial parameter; $Z e / p c$ is also constant, and then finally we get

$\rho A_{\phi}=$ const.

It is easy to show that Eq. (20) is the equation of the magnetic field line in cylindrical coordinates assuming axial symmetry. Thus, in the allowed region of motion, the trapped particle moves around a magnetic field line; this equation is also the equation of the guiding center of the particle. The particle oscillates between the two ends of the allowed region where the mirror points are located. As an example, let us consider the case of a dipole field. Using Eqs. (8), (17) and vector potential Eq. (12) for the boundaries of the allowed region, we get

$\frac{v_{\phi}}{v}=\frac{2 \gamma}{\rho}-\frac{\rho}{r^{3}}$.

If we consider the case where $v_{\phi} / v=0$, then taking into account the fact that in spherical coordinates $\rho=r \sin \theta$, we rewrite Eq. (21):

$r=\frac{1}{2 \gamma} \sin ^{2} \theta$.

If we consider the motion in the equatorial plane of a dipole, then $\theta=90^{\circ}$, and we get

$r_{\mathrm{eq}}=\frac{1}{2 \gamma}$.

If we put Eq. (23) into Eq. (22), we get the famous equation of the dipole field line of the planet:

$r=r_{\mathrm{eq}} \sin ^{2} \theta$.

The boundaries of the allowed region of motion are determined by the equality to \pm 1 in Eq. (21), and as we have just shown, the center of this region corresponds to the position of the guiding center of the particle.

To describe the particle trajectory in the whole space, we can change from cylindrical coordinates $(\rho, \phi, z, \dot{\rho}, \rho \dot{\phi}, \dot{z})$ to phase-space coordinates $\left(\rho_{0}, \phi_{0}, z_{0}, v, P_{\phi}, \rho_{0} \dot{\phi_{0}}\right)$. Here, the three variables $\left(\rho_{0}, \phi_{0}, z_{0}\right)$ fix the starting point, and the other three variables $\left(v-\right.$ velocity; $P_{\phi}-$ generalized moment of a particle; $\rho_{0} \dot{\phi}_{0}-$ an azimuthal component of the velocity, which determines an initial phase of Larmor rotation) fix the initial particle velocity vector. Thus, two integrals of motion $\left(v\right.$ and $P_{\phi}$ ) fix a bundle of trajectories for particles with the same energy and the same azimuthal component of generalized momentum. All trajectories with different phases of Larmor rotation $\phi$ (which changes from 0 to $2 \pi$ ) form a tube 


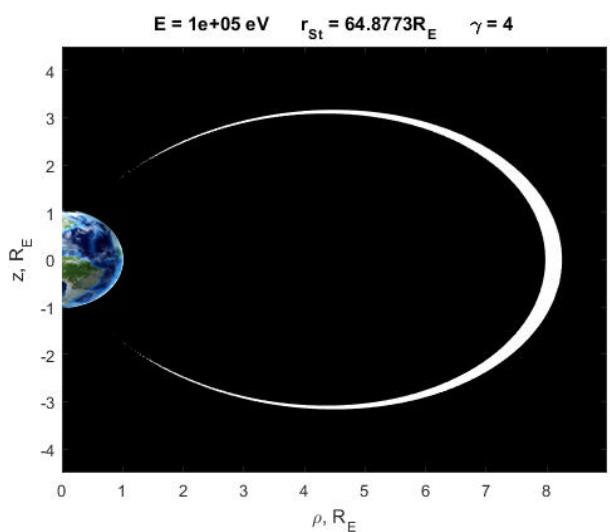

(a)

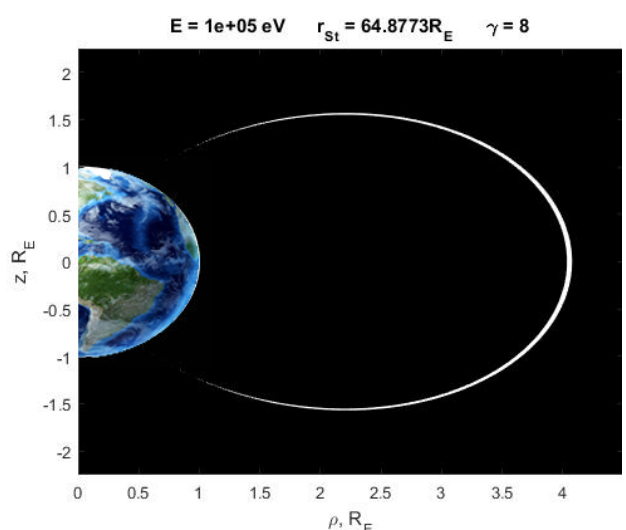

(b)

Figure 2. Allowed regions of motion of a $100 \mathrm{keV}$ proton for two cases: (a) $\gamma=4$ and (b) $\gamma=8$. It can be seen that for $\gamma=4$, the allowed region of particle motion crosses the equatorial plane at a distance of $8 R_{\mathrm{E}}$, and for $\gamma=8$ at $4 R_{\mathrm{E}}$. Thus, with increasing $\gamma$, the particle comes closer to the Earth. The allowed region of motion is highlighted in white.

around the particle's guiding center field line, which is an allowed region of motion.

According to Eq. (23), the larger the $\gamma$, the closer a particle is located to the Earth. This can be explained in the following way. In Eqs. (8) and (21) two terms on the right-hand side represent the effect of the centrifugal force and magnetic field, respectively, which hold the particle away from the axis. The closer the particle is to the Earth ( $\rho$ decreases), the larger the second term (magnetic field) on the right-hand side is, and, accordingly, the larger the first term must be to compensate for the second. Thus, as the particle approaches the Earth, with decreasing $\rho$, its parameter $\gamma$ must increase.

This situation is shown in Fig. 2, where the allowed motion regions for the $100 \mathrm{keV}$ proton with different parameters $\gamma$ are shown. The larger $\gamma$ is, the closer the allowed region of motion shifts to the Earth.

The allowed region shown in Fig. 2a and b is not the only one and unique for the particle - it can be trapped either closer to the planet or farther, but with different initial parameters, which determines its trajectory and allowed region of motion. In order for a particle to get into the currently forbidden region, the parameter $\gamma$ must change as a result of some process occurring in the magnetosphere. Thus the whole allowed region of motion is determined by a change in $\gamma$ (or $P_{\phi}$ ) from 0 to $+\infty$. The size of the allowed region of motion equals 2 times the Larmor radius of a particle at a specific point. For the case of Fig. 2a, the Larmor radius is $754 \mathrm{~km}$ or 0.118 Earth radii, and for the case of Fig. 2b, it is $94 \mathrm{~km}$ or 0.015 Earth radii.

To find $\gamma$ corresponding to a particle with a certain energy located on a certain $L$ shell, it is necessary to use Eq. (23). In Fig. 3 we show the dependence of $\gamma$ on a particle's energy for different $L$ shells. We can see that the closer the particle's $L$ shell to the Earth, the higher $\gamma$ it should have. Also, if a particle's energy is rather low, $\gamma$ should be changed greatly to move a particle from a higher $L$ shell to a lower $L$ shell.

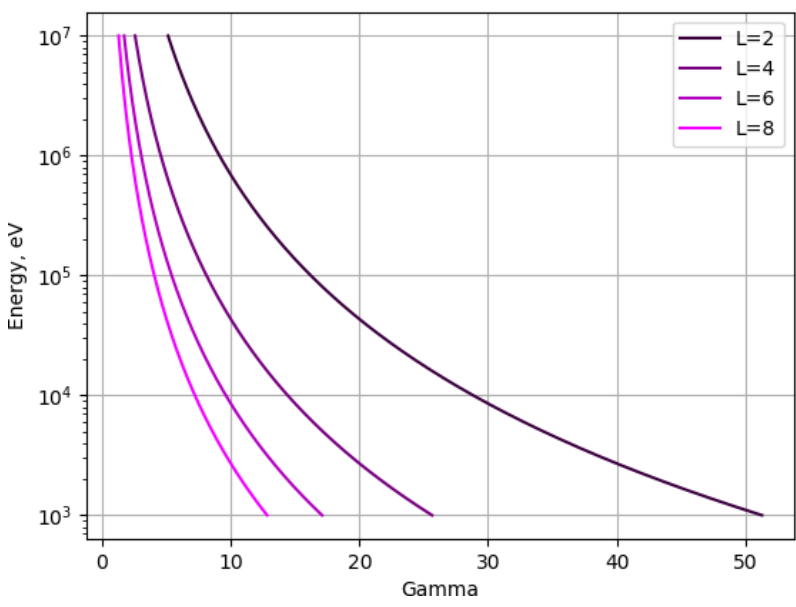

Figure 3. Dependence of Størmer's parameter $\gamma$ on the particle's energy for four different $L$ shells.

In the original Størmer analysis the magnetic field is stationary and a particle does not receive energy from outside, so the parameter $\gamma$ will remain constant. In reality, the solar wind flow past the magnetosphere generates a large-scale long-standing convection electric field in it, directed from dawn to dusk under the southward-oriented IMF. As a result, the $\boldsymbol{E} \times \boldsymbol{B}$ drift occurs in the magnetosphere, with the particles moving from the magnetotail towards the Earth, thus supplying the radiation belts and ring current with particles. In addition to the large-scale potential electric fields that appear in the nightside magnetosphere due to the prolonged southward-oriented IMF, the impulsive-induced electric fields (which can also transport particles into the inner magnetosphere) can arise due to the magnetic field reconfigurations during substorms (Daglis, 2001). These two particles' transport and acceleration mechanisms can act simultaneously (Ebihara and Ejiri, 2003) and are responsible for 
the injection of particles into the inner magnetosphere and thus for the change in parameter $\gamma$. It will increase when approaching the planet due to the increase in the magnetic field strength, as mentioned above.

\section{Modeling of Størmer's allowed and forbidden regions taking into account the ring current}

In modeling, we assumed the following.

1. The Earth's magnetic field is constant and has only a dipole component due to the axial symmetry of the problem. This is quite reasonable, because (1) the dipole magnetic field is responsible for the basic motion of the ring current particles, that is, the bounce and drift motion, and (2) the higher degree of the coefficient becomes dominant at a radial distance of less than $1.5 R_{\mathrm{E}}$ or above the South Atlantic and South African areas.

2. The ring current has the form of an infinitely thin ring in the equatorial plane at a distance of $4 R_{\mathrm{E}}$ from the center of the Earth. This value was chosen in accordance with the spatial dimensions of the ring current in the equatorial plane and with average values of the maximum of the ring current density (Kovtyukh and Panasyuk, 2008; Prölss, 2004). We assume that not all of the ring current particles are concentrated in the filamentary ring, but that the magnetic field, created by the real ring current, is the same as created by the filamentary ring with current.

3. The $b_{z}$ component of the homogeneous external field is $-15 \mathrm{nT}$ and describes the field of distant sources: the magnetopause current's field (positive 20-100 nT), the field of tail currents (negative 10-200 nT) and the penetrating interplanetary field (about $1-20 \mathrm{nT}$ of an arbitrary sign).

4. Charged particles in both the radiation belt and ring current are protons $\left(\mathrm{H}^{+}\right)$.

5. Radiation belt particles with energies of 1 and $100 \mathrm{MeV}$.

6. Ring current particles with energies of 10 and $100 \mathrm{keV}$.

For particles with different values of $\gamma$ and current strengths' $I$, different patterns of the regions' boundaries are obtained. We know that in reality the particles of the ring current are distributed up to $\sim 8 R_{\mathrm{E}}$ (see Sect. 2). Using Eq. (23), we choose those values of $\gamma$ that describe particles of different energies at a distance $L=8$. This distance will be considered the outer edge of the ring current. Having set the $\gamma$ and energy of the particle (initial parameters), we begin to change the current strength and look what happens with the allowed regions of motion of the particle.

All the following figures show the meridional cross sections of the near-Earth region; the allowed regions of motion are highlighted in white, forbidden in black. Magnetic field lines are highlighted in cyan. Coordinates are measured in the Earth radii. The spatial configuration of forbidden regions is obtained by rotating the meridional sections around the $z$ axis. Particles cannot cross the forbidden region and, consequently, free particles cannot penetrate into the internal allowed region. Likewise, particles that somehow find themselves in an internal allowed region in the ideal case are permanently trapped.

At first we consider the energies of the ring current protons of 10 and $100 \mathrm{keV}$ (Figs. 4, 5 and 6).

As can be seen from Figs. 4 to 6, with increasing ring current strength, two initially separated (at $I=0$ A) allowed regions of motion become connected. Moreover, for particles with energies of $100 \mathrm{keV}$, the escape path from the inner trapping region emerges earlier than for particles with an energy of $10 \mathrm{keV}$, at a current strength of $9.17 \mathrm{MA}$ (Fig. 5b). When the current reaches $10.28 \mathrm{MA}$, particles with energy of $10 \mathrm{keV}$ also get the opportunity to leave the trapping region (Fig. 6a). As a result, particles of the outer edge of the ring current with different energies are able to leave the trapping region area.

The obtained current strengths are consistent with the observations (see Sect. 2), and the obtained Dst index values for these current strength magnitudes are 226 and $253 \mathrm{nT}$, which are not unique and usually characterize large storms. However, we must remember that the Dst index was calculated by Eq. (2) for an infinitely thin ring with current; i.e., it was assumed that all particles are located in this ring. In reality the situation is different: the particles are distributed in the toroidal volume around the Earth over a large range of distances; in addition, for the ring current and homogeneous magnetic field the condition of axial symmetry is not satisfied, so the calculation of the real Dst index is much more complicated. Nevertheless, our analysis makes sense, since it gives an upper limit on the maximum ring current strength that retains the trapping regions. Taking into account the asymmetry will lower this threshold, since the particles will have an opportunity to leave the trapping region in the nightside, going to infinity through the geomagnetic tail or leaving the magnetosphere through the daytime magnetopause. In this case, the "bridge" between the trapping region and infinity opens the possibility of injection of the plasma sheet particles into the ring current region with simultaneous acceleration and an increase in the trapped particles' number. However, the obtained magnitudes may probably indicate that the other loss mechanisms begin to act earlier than the mechanism suggested in this paper. The balance of sources and losses describes the observed dynamics of the magnetic storm.

Now let us consider what happens to the protons of the radiation belts (with energies of 1 and $100 \mathrm{MeV}$ ) after increase in the ring current magnitude (Figs. 7-8) for the same magnitudes of the currents chosen for Figs. 4-6. The most distant protons of the radiation belt are located at a distance of $\sim 2.5 R_{\mathrm{E}}$ (see Sect. 2). 


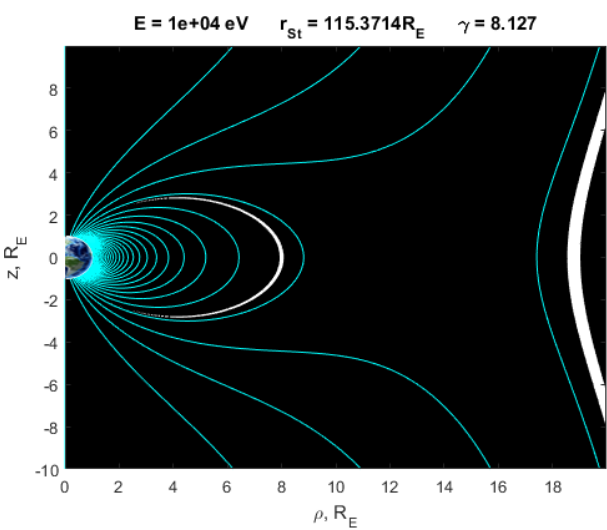

(a)

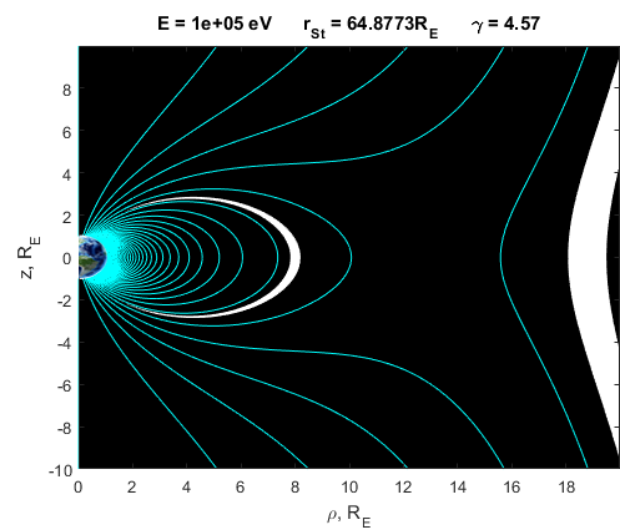

(b)

Figure 4. The ring current strength $I$ is $0 \mathrm{MA}$. (a) $10 \mathrm{keV}$; (b) $100 \mathrm{keV}$.

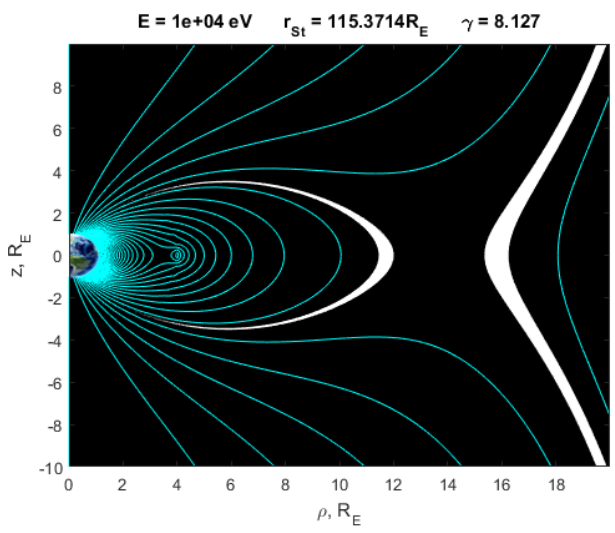

(a)

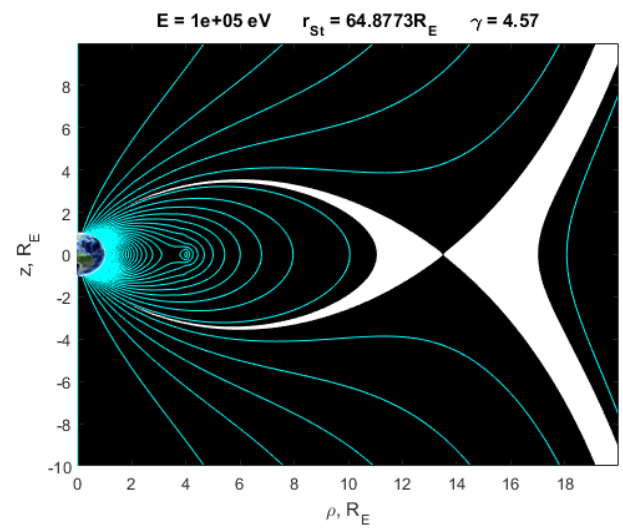

(b)

Figure 5. The ring current strength $I$ is $9.17 \mathrm{MA}$. (a) $10 \mathrm{keV}$; (b) $100 \mathrm{keV}$.

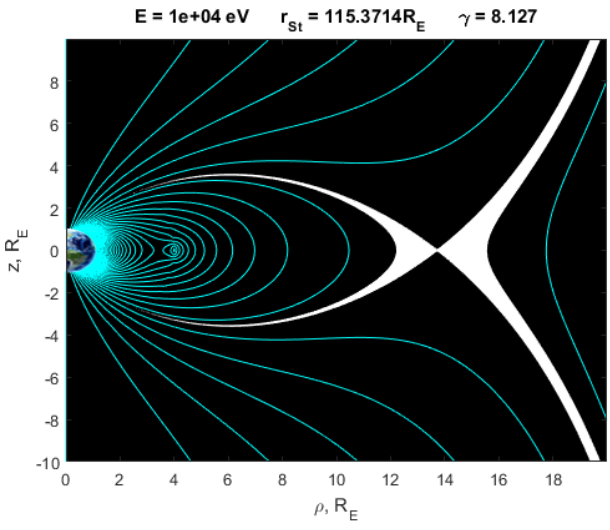

(a)

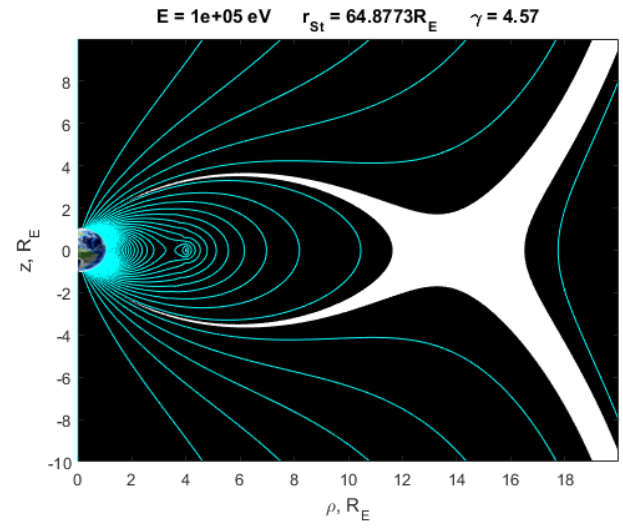

(b)

Figure 6. The ring current strength $I$ is $10.28 \mathrm{MA}$. (a) $10 \mathrm{keV}$; (b) $100 \mathrm{keV}$.

As can be seen from Figs. 7 to 8, the internal trapping regions slightly increase in size with the ring current strength enhancement. Thus particle fluxes of the radiation belts will decrease due to the increase in the spatial dimensions of the allowed region, as observed on the Earth during magnetic storms (Kovtyukh and Panasyuk, 2008). At the same time, the depression of the Earth's magnetic field is still not large enough for the particles of the radiation belt to be able to leave the trapping region. This is consistent with the fact that particles can exist in the radiation belts for a long time 


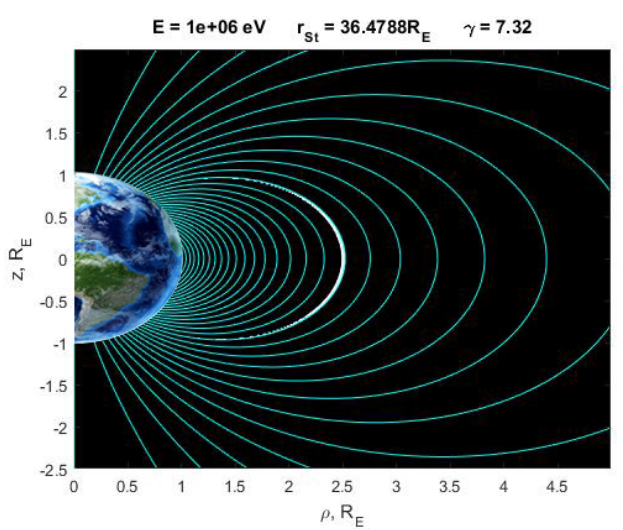

(a)

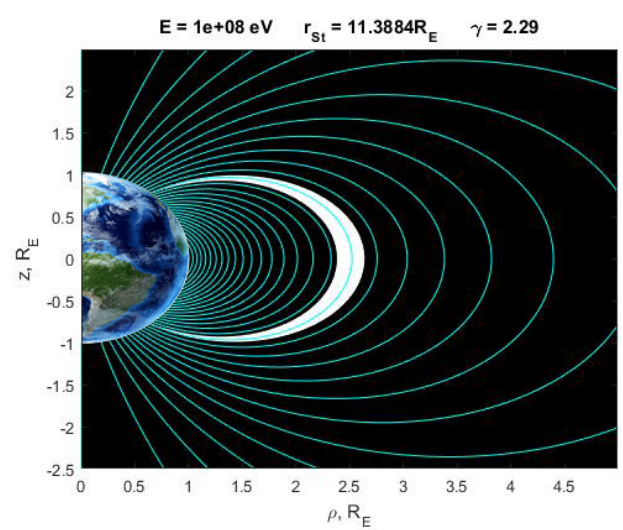

(b)

Figure 7. The ring current strength $I$ is $0 \mathrm{MA}$. (a) $1 \mathrm{MeV}$; (b) $100 \mathrm{MeV}$.

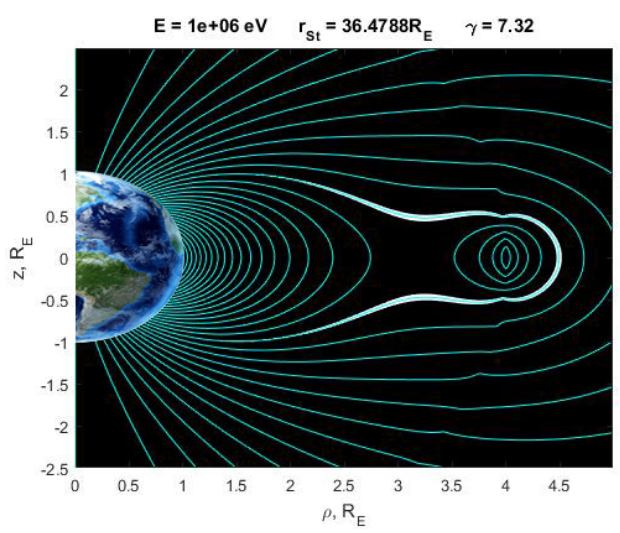

(a)

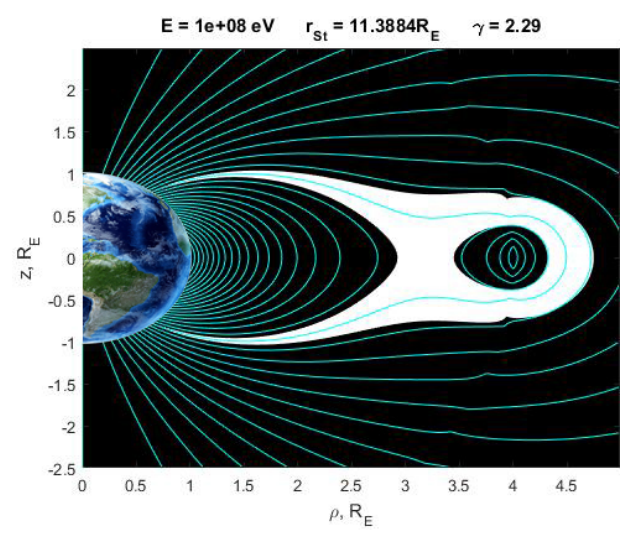

(b)

Figure 8. The ring current strength $I$ is $10.28 \mathrm{MA}$. (a) $1 \mathrm{MeV}$; (b) $100 \mathrm{MeV}$.

(Prölss, 2004). We should mention noticeable artifacts in the field lines' structure associated with the mathematical expression of the ring current vector potential.

However, the geometry of the allowed-forbidden regions and the current strength at which the inner and outer Størmer trapping regions get connected strongly depend on two parameters - the external magnetic field $b_{z}$ and the radius $a$ of the current ring. As an example, we consider the region of motion of the $10 \mathrm{keV}$ proton in the presence of the ring current of 9.17 MA for three magnitudes of the external field $b_{z}$ : $-10,-15$, and $-20 \mathrm{nT}$ (Fig. 9a-c).

We consider an initial state for a trapped particle under external field $b_{z}=-10 \mathrm{nT}$ (Fig. 9b). When $b_{z}$ decreases by $5 \mathrm{nT}$, more particles get the opportunity to leave the trapping region much earlier (Fig. 9c). If, on the other hand, $b_{z}$ increases by $5 \mathrm{nT}$ (Fig. 9a), the trapping of particles becomes even stronger - they move closer to the Earth and the outer allowed region of motion moves away from the Earth. This means that the magnitude of the external field $b_{z}$ strongly controls the trapping condition for particles in our problem.
Now let us consider how the configuration of the particles' allowed regions of motion with the same parameters varies for different radii $a$ of the current ring. Again we consider a proton with an energy of $10 \mathrm{keV}$ and a current strength equal to $9.17 \mathrm{MA}$; for $a$ we take three different values $-3,4$ and $5 R_{\mathrm{E}}$ (Fig. 10a-c).

One can see that Figs. 9 and 10 are quite similar. Increase in the external field $b_{z}$ leads to the same result as the decrease in the ring current radius $a$ and to the stronger trapping of particles (Figs. 9a, 10a). Decrease in $b_{z}$ (Fig. 9c) leads to the break of the trapping region; the same happens when the radius $a$ of the ring current increases (Fig. 9c). As the field inside the current ring decreases, and outside increases, we see a logical result in Fig. 10a-c. For $a=4 R_{\mathrm{E}}$ (Fig. 10b), particles will be in the region of a stronger field (ring current field plus dipole) than for $a=5 R_{\mathrm{E}}$ (Fig. $10 \mathrm{c}$ ) - in this case the field at $L=8 R_{\mathrm{E}}$ is weaker. Therefore, the larger the radius of the model ring with current, the faster the particles at the edge of the real ring current get the opportunity to leave the trapping region. Conversely, when the radius of the current ring decreases (Fig. 10a), the field magnitude beyond the distance 


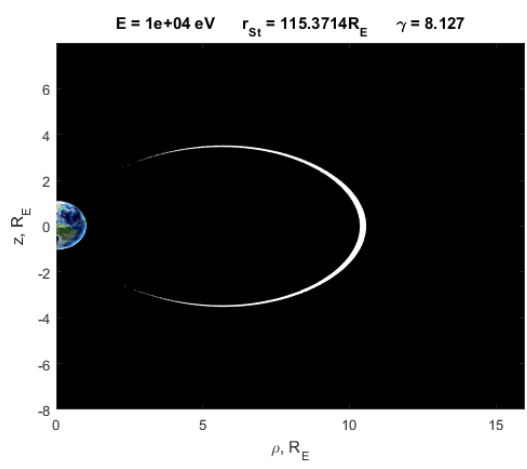

(a)

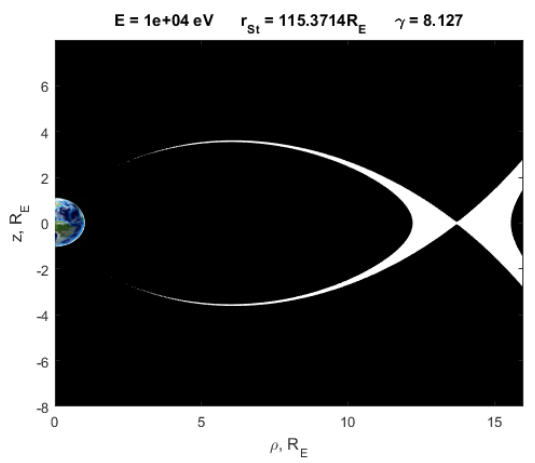

(b)

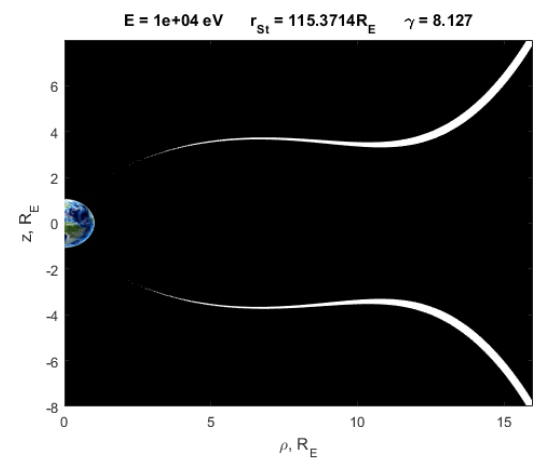

(c)

Figure 9. The strength of the ring current is $9.17 \mathrm{MA}$; the energy of the proton is $10 \mathrm{keV}$. (a) $b_{z}=-10 \mathrm{nT}$, (b) $b_{z}=-15 \mathrm{nT}$, and (c) $b_{z}=$ $-20 \mathrm{nT}$

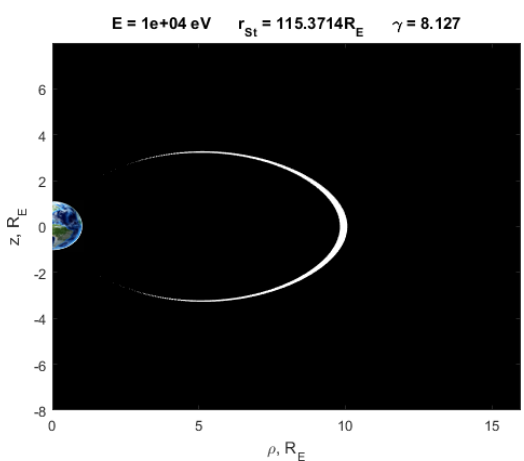

(a)

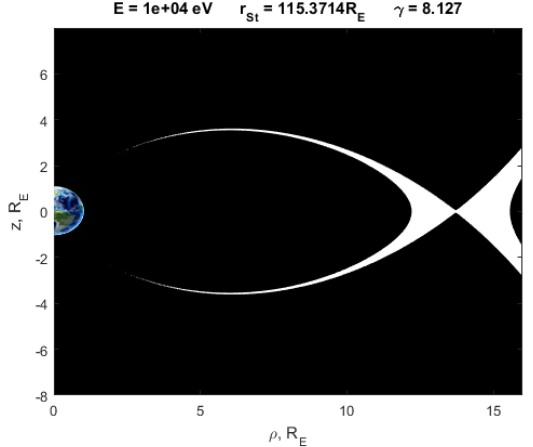

(b)

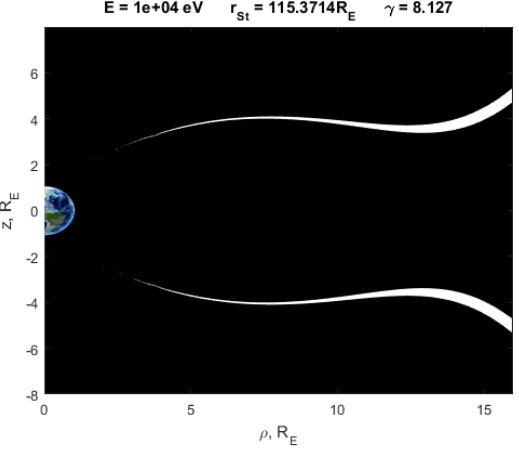

(c)

Figure 10. The strength of the ring current is $9.17 \mathrm{MA}$; the particle energy is $10 \mathrm{keV}$. (a) $a=3 R_{\mathrm{E}}$, (b) $a=4 R_{\mathrm{E}}$, and (c) $a=5 R_{\mathrm{E}}$.

$a$ becomes larger and the particles are trapped more strongly by the magnetic field. Thus, the topology of the allowedforbidden regions of motion and the critical value of the Dst index in our problem also strongly depends on the radius $a$ of the ring with the current.

Størmer analysis is not a self-consistent approximation; the magnetic field in the problem is axially symmetric and specified - independent of the trapped particles' number. When searching for the critical value of the ring current, we set the distance based on the available observations. Nevertheless, for a complete solution of the problem it would be useful to have a generalization of the Størmer analysis to the case of a self-consistent field. We will try to show how to come to this solution by the method of successive approximations. We will calculate the threshold current for particles with fixed energy at a given distance, changing the external field. Afterwards, we will calculate the threshold current for particles with fixed energy with a given external field, changing the radius $a$ of the ring current. The distance at which the ring current is concentrated will be determined from the actual external field and the observed field at the origin. Having a Dst magnitude, the Dessler-Parker-Sckopke relation (Dessler and Parker, 1959; Sckopke, 1966) can be used to calculate the total ion energy content in the dipole magnetic
Table 1. Critical strength of a ring current for different magnitudes of the external field $b_{z}$.

\begin{tabular}{lrr|rr}
\hline$b_{z}, \mathrm{nT}$ & \multicolumn{2}{c|}{$10 \mathrm{keV}$ proton } & \multicolumn{2}{c}{$100 \mathrm{keV}$ proton } \\
\cline { 2 - 5 } & $I_{\mathrm{cr}}$, MA & $r_{\mathrm{cr}}, R_{\mathrm{E}}$ & $I_{\mathrm{cr}}$, MA & $r_{\mathrm{cr}}, R_{\mathrm{E}}$ \\
\hline 0 & 1952.7 & 832 & 591.2 & 263.1 \\
-5 & 35.9 & 22.7 & 32.9 & 22.1 \\
-10 & 17.7 & 16.5 & 16.1 & 16.1 \\
-15 & 10.3 & 13.7 & 9.1 & 13.5 \\
-20 & 6.3 & 12.1 & 5.4 & 11.9 \\
\hline
\end{tabular}

field and hence the maximum number of particles that can be trapped by the dipole magnetic field.

Let us consider a proton with energies of 10 and $100 \mathrm{keV}$ at $L=8 R_{\mathrm{E}}$. For each $b_{z}$ magnitude we will search for the current strength magnitude at which the forbidden region between two allowed regions breaks (and the distance at which this link happens). The results are shown in Table 1.

It can be seen from Table 1 that the field $b_{z}$ is very important for determining the critical ring current. The stronger the external field, the faster the ring current breaks and at a lower strength. When $b_{z}$ vanishes, enormous current strength 
Table 2. Critical strength of a ring current for different ring radii $a$.

\begin{tabular}{lrr|rr}
\hline$a, R_{\mathrm{E}}$ & \multicolumn{2}{c|}{$10 \mathrm{keV}$ proton } & \multicolumn{2}{c}{$100 \mathrm{keV}$ proton } \\
\cline { 2 - 5 } & $I_{\mathrm{cr}}, \mathrm{MA}$ & $r_{\mathrm{cr}}, R_{\mathrm{E}}$ & $I_{\mathrm{cr}}, \mathrm{MA}$ & $r_{\mathrm{cr}}, R_{\mathrm{E}}$ \\
\hline 3 & 31.8 & 16.4 & 28.9 & 16.1 \\
4 & 17.7 & 16.5 & 16.1 & 16.1 \\
5 & 11.2 & 16.5 & 10.2 & 16.2 \\
6 & 7.6 & 16.6 & 6.9 & 16.2 \\
7 & 5.5 & 16.6 & 4.9 & 16.3 \\
\hline
\end{tabular}

is needed to break the forbidden region, which will be even larger for a case with an external field with a positive $z$ component. One can also see that for particles with different energies, the point $r_{\mathrm{cr}}$, where the break occurs, is displaced closer to the Earth and gradually converges to a certain value.

Let us also consider the influence of the radius $a$ of the ring with current on the critical strength of the current. We again take a proton with energies of 10 and $100 \mathrm{keV}$ at $L=8 R_{\mathrm{E}}$ at $b_{z}=-10 \mathrm{nT}$. For each value of the radius of the ring with current, we will search for the current strength magnitude at which the forbidden region between two allowed regions breaks for the protons of the ring current under consideration.

As one can see from Table 2, the radius of the selected ring has an influence on the critical current strength: for example, for $a=3 R_{\mathrm{E}}$ and $a=5 R_{\mathrm{E}}$, the critical magnitude of the ring current differs by a factor of 3 . Although the effect of the external field on the current strength is much larger, it is obvious that both parameters $a$ and $b_{z}$ influence the critical ring current strength, which can exist, and, therefore, the Dst index.

The change in the radius $a$ of the ring current, as can be seen from Table 2, has almost no effect on the distance $r_{\mathrm{cr}}$ to the point, where the forbidden region breaks. Thus, having the constant $b_{z}$ field, there will be a direct dependence between $I_{\mathrm{cr}}$ and $a$ (Table 2). Now, having dependence between $b_{z}$ and $I_{\text {cr }}$ (from Table 1), it becomes possible to calculate the ring current radius $a$ for a given $b_{z}$ value.

\section{Conclusions}

As a result of particles' injection into the inner magnetosphere, their population and consequently the ring current strength increase. Størmer's analysis shows that at a certain point, when the ring current strength reaches a critical magnitude, the forbidden region, which separates the internal and external allowed regions, disappears, and thus particles get the opportunity to leave the internal trapping region. As a result, the number of particles, which comprise the ring current, and consequently the current strength, begin to decrease. In the limit in this case, the particle density near the dipole and at infinity must be the same. The critical magnitude of the ring current's strength depends on the energy of the parti- cles composing the ring current, but at a certain moment the maximum strength is reached, when particles even with the lowest energy are able to leave the trapping region. The maximum possible number of particles that can be trapped in a dipole field is determined by a number of particles, forming a ring current, which changes the "connectivity" of Størmer's regions. For the doubly connected geometry of Størmer's regions (when internal and external allowed regions of motion are not connected), the particles' flux at infinity and the flux of trapped particles close to the Earth can differ arbitrarily. The mixing of passing (coming and going to infinity) and trapped particles does not occur. Therefore, it becomes possible to form regions of trapped radiation, the fluxes of which are determined by the injection and loss mechanisms and are not associated with particles' fluxes at infinity. The deformation of Størmer's regions by the self-consistent field of the trapped radiation (ring current) leads to the formation of connected allowed regions of motion, and in this case the particles' fluxes in the allowed region are uniquely determined by the particles' flux and distribution over the pitch angles at infinity. We come to the conclusion that the ring current strength has a finite upper limit. At a certain current strength magnitude, the inner trapping region becomes connected to the outer region associated with infinity and further accumulation of particles in the internal trapping region is impossible. At present, there are several recognized main processes, leading to the ring current decay. We showed that a new mechanism, described in the paper, can also be added to these mechanisms. This mechanism leads to the limiting of the ring current strength during a magnetic storm irrespective of the particle loss mechanism from the internal allowed trapping region.

Størmer's analysis shows that the existence of radiation belts follows from the existence of a geomagnetic field. Particles with particle energies of radiation belts and with different parameters $\gamma$ are trapped at different $L$ shells, depending on Størmer's parameter $\gamma$. In addition to the analysis of the critical strength of the ring current, we tried to explain how Størmer's parameter $\gamma$ is defined and changed. Its change leads to the displacement of the inner trapping region closer to the planet, and, at the same time, to the region's size decrease. The parameter $\gamma$ of a particle can be changed by several mechanisms, for example by $\boldsymbol{E} \times \boldsymbol{B}$ drift, which moves particles to the inner magnetosphere.

Data availability. No datasets were used in the preparation of this paper. All the physical quantities' values are public knowledge.

Author contributions. ASL and IIA were responsible for the planning, coordination, revisions, and responses to the referees. ASL was responsible for the writing of the entire paper and IIA proposed the original idea; IVT was responsible for calculations and plotting the corresponding results. 
Competing interests. The authors declare that they have no conflict of interest.

Acknowledgements. The work was supported by the Ministry of Science and Higher Education of the Russian Federation (grant RFMEFI61617X0084).

Financial support. The work was supported by the Ministry of Science and Higher Education of the Russian Federation (grant RFMEFI61617X0084).

Review statement. This paper was edited by Elias Roussos and reviewed by two anonymous referees.

\section{References}

Alexeev, I. I.: Energy flux in the Earth's magnetosphere: Storm - substorm relationship, Space Sci. Rev., 107, 141-148, https://doi.org/10.1023/A:1025519622160, 2003.

Alexeev, I. I., Belenkaya, E. S., Kalegaev, V. V., Feldstein, Y. I., and Grafe, A.: Magnetic storms and magnetotail currents, J. Geophys. Res.-Space, 101, 7737-7747, https://doi.org/10.1029/95JA03509, 1996.

Baumjohann, W., Blanc, M., Fedorov, A., and Glassmeier, K.-H.: Current Systems in Planetary Magnetospheres and Ionospheres, Space Sci. Rev., 152, 99-134, https://doi.org/10.1007/s11214010-9629-z, 2010.

Daglis, I.: The storm-time ring current, Space Sci. Rev., 98, 343363, https://doi.org/10.1023/A:1013873329054, 2001.

Daglis, I. A., Thorne, R. M., Baumjohann, W., and Orsini, S.: The terrestrial ring current: Origin, formation, and decay, Rev. Geophys., 37, 407-438, https://doi.org/10.1029/1999RG900009, 1999.

Dessler, A. J. and Parker, E. N.: Hydromagnetic theory of geomagnetic storms, J. Geophys. Res., 64, 2239-2252, https://doi.org/10.1029/JZ064i012p02239, 1959.

Ebihara, Y. and Ejiri, M.: Modeling of solar wind control of the ring current buildup: A case study of the magnetic storms in April 1997, Geophys. Res. Lett., 25, 3751-3754, https://doi.org/10.1029/1998GL900006, 1998.

Ebihara, Y. and Ejiri, M.: Numerical Simulation of the Ring Current: Review, Space Sci. Rev., 105, 377-452, https://doi.org/10.1023/A:1023905607888, 2003.
Jackson, J. D.: Classical Electrodynamics, John Wiley \& Sons Inc., New York, USA, 1963.

Kovtyukh, A. S. and Panasyuk, M. I.: The storm-time ring current, in: Plasma heliogeophysics, edited by: Zeleny, L. M. and Veselovsky, I. S., Vol. 1, chap. 4, 534-552, Fizmatlit Moscow, 2008.

Kozyra, J. U. and Liemohn, M. W.: Ring Current Energy Input and Decay, Space Sci. Rev., 109, 105-131, https://doi.org/10.1023/B:SPAC.0000007516.10433.ad, 2003.

Landau, L. and Lifshitz, E.: Mechanics, Butterworth-Heinemann, 3rd Edn., Oxford, UK, 1976.

Landau, L., Lifshitz, E., and Pitaevskii, L.: Electrodynamics of Continuous Media, Butterworth-Heinemann, 2nd Edn., Oxford, UK, 1982.

Lemaire, J.: The effect of a southward interplanetary magnetic field on Störmer's allowed regions, Adv. Space Res., 31, 1131-1153, https://doi.org/10.1016/S0273-1177(03)00099-1, 2003.

Morozov, A. and Soloviyov, L.: Magnetic field geometry, in: Voprosy Teorii Plazmy (Problems in Plasma Theory), edited by: Leontovich, M. A., Vol. 2, chap. 1, Atomizdat, Moscow, USSR, 1963.

Prölss, G.: Physics of the Earth's Space Environment, SpringerVerlag, Berlin Heidelberg, https://doi.org/10.1007/978-3-64297123-5, 2004.

Ray, E. C.: Effects of a Ring Current on Cosmic Radiation, Phys. Rev., 101, 1142-1148, https://doi.org/10.1103/PhysRev.101.1142, 1956.

Sckopke, N.: A general relation between the energy of trapped particles and the disturbance field near the Earth, J. Geophys. Res., 71, 3125-3130, https://doi.org/10.1029/JZ071i013p03125, 1966.

Shebalin, J. V.: Størmer regions for axisymmetric magnetic multipole fields, Phys. Plasmas, 11, 3472-3482, https://doi.org/10.1063/1.1752931, 2004.

Størmer, C.: Sur les trajectoires des corpuscules électrisés dans l'espace sous l'action du magnétisme terrestre, avec application aux aurores boréales, Radium (Paris), 9, 395-399, https://doi.org/10.1051/radium:01912009011039501, 1912.

Størmer, C.: The Polar Aurora, Clarendon, Oxford, UK, 1955.

Treiman, S. B.: Effect of Equatorial Ring Current on Cosmic-Ray Intensity, Phys. Rev., 89, 130-133, https://doi.org/10.1103/PhysRev.89.130, 1953.

Turner, N. E., Baker, D. N., Pulkkinen, T. I., and McPherron, R. L.: Evaluation of the tail current contribution to Dst, J. Geophys. Res.-Space, 105, 5431-5439, https://doi.org/10.1029/1999JA000248, 2000. 